\title{
Hipertensión arterial, diabetes mellitus y factores de riesgo cardiovasculares en trabajadores de una plaza de mercado de un municipio de Antioquia, Colombia, 2017-2018*
}

High blood pressure, diabetes mellitus, and cardiovascular risk factors in workers in a market square in a municipality of Antioquia, Colombia, 2017-2018

\section{Hipertensão arterial, diabetes mellitus e fatores de risco cardiovascular em trabalhadores de uma praca da feira em um município de Antioquia, Colômbia, 2017-2018}

Recibido: 03 de abril de 2020. Aceptado: 16 de septiembre de 2020. Publicado: 31 de mayo de 2021.

DOI: https://doi.org/10.11144/Javeriana.rgps20.hadm

\author{
Daniel Vasquez ${ }^{a}$ \\ Universidad CES, Colombia \\ ORCID: https://orcid.org/0000-0002-2586-162X \\ María Osley Garzón-Duque \\ Universidad CES, Colombia \\ ORCID: https://orcid.org/0000-0001-7261-3146
}

\begin{abstract}
Para citar este artículo: Vasquez D, Garzón-Duque MO. Hipertensión arterial, Diabetes Mellitus y factores de riesgo cardiovasculares en trabajadores de una plaza de mercado de un municipio de Antioquia, Colombia, 2017-2018. Rev Gerenc Polit Salud. 2021;20. https://doi.org/10.11144/ Javeriana.rgps20.hadm
\end{abstract}

* Artículo de investigación.

a Autor de correspondencia. Correo electrónico: danielvasquezb96@gmail.com 


\section{Resumen}

Introducción. Enfermedades como la diabetes mellitus y la hipertensión arterial son cada vez más frecuentes a nivel mundial con importantes costos sociales asociados. Objetivo. Evaluar la prevalencia de hipertensión y diabetes en los trabajadores de la plaza de mercado de un municipio de Antioquia, Colombia y los factores de riesgo asociados. Metodología. Se llevó a cabo un estudio observacional de tipo transversal analítico en un censo de 194 trabajadores de los cuales 152 tuvieron evaluación médico-laboral y encuesta de datos sociodemográficos y laborales. Se hizo una estandarización previa de los evaluadores con control de calidad del dato y análisis univariado y bivariado de los datos con IC 95\%. Resultados. El 57\% era mujer, 73,4\% ganaba menos de un salario mínimo mensual legal vigente, $65,8 \%$ laboraba entre 9 y 12 horas y $11,9 \%$ entre 13 y 18 horas al día. El $62,4 \%$ no realizaba actividad física y $67,0 \%$ prefería los alimentos fritos. Un 20,9\% tenía hipertensión arterial y se asoció con el estado civil, edad, antigüedad en el oficio, presión sistólica al momento de la evaluación, antecedente familiar y sexo. Un $9,2 \%$ tenía diabetes mellitus y esto se asoció con el estado civil, edad y presión sistólica al momento de la evaluación. Conclusiones. Se encuentra una población trabajadora informal con alta carga de enfermedad y de factores de riesgo que evidencian la necesidad de políticas públicas y programas enfocados específicamente en sus riesgos y estilos de vida.

Palabras clave: Sector informal, hipertensión, diabetes mellitus, mercado de trabajo.

\section{Abstract}

Introduction. Diseases like diabetes mellitus and high blood pressure are increasingly frequent worldwide, with significant associated social costs. Objective. To evaluate the prevalence of high blood pressure and diabetes in workers in the marketplace of a municipality in Antioquia, Colombia, and the associated risk factors. Methodology. An analytical cross-sectional observational study was conducted on a census of 194 workers, of which 152 had a medicallabor assessment and a survey of socio-demographic and labor data. A previous standardization of the evaluators was made with data quality control and univariate and bivariate data analysis, with IC $95 \%$. Results. 57\% were women, $73.4 \%$ earned less than the current legal monthly minimum wage; $65.8 \%$ worked between 9 and 12 hours, and $11.9 \%$ between 13 and 18 hours per day. 62.4\% don't engage in physical activity, and $67.0 \%$ prefer fried food. $20.9 \%$ had high blood pressure that was associated with marital status, age, seniority in the trade, systolic pressure at the time of evaluation, family history, and gender. $9.2 \%$ had diabetes mellitus, and this was associated with marital status, age, and systolic pressure at the time of evaluation. Conclusions. There is an informal working population with a high burden of disease and risk factors, which shows the need for public policies and programs specifically focused on their risk and lifestyles.

Keywords: Informal sector, high blood pressure, diabetes mellitus, work market.

\section{Resumo}

Introdução. Doenças como diabetes mellitus e hipertensão arterial são cada vez mais frequentes em todo o mundo com importantes custos sociais associados. Objetivo. Avaliar a prevalência de hipertensão e diabetes em trabalhadores da praça da feira de um município de Antioquia, Colômbia e os fatores de risco associados. Metodologia. Foi realizado um estudo observacional de ordem transversal analítica em um censo de 194 trabalhadores, dos quais 152 tiveram avaliação médico-laboral e levantamento de dados sociodemográficos e trabalhistas. Os avaliadores foram previamente padronizados com controle de qualidade dos dados e análise univariada e bivariada dos dados com IC95\%. Resultados. 57\% era mulher, 73,4\% ganhava menos de um salário mínimo mensal legal vigente, $65,8 \%$ trabalhava entre 9 e 12 horas e $11,9 \%$ entre 13 e 18 horas diárias; $62,4 \%$ não praticava atividade física e $67,0 \%$ prefere alimentos fritos. 20,9\% apresentava hipertensão arterial e estava associada a estado civil, idade, tempo de serviço, pressão sistólica no momento da avaliação, antecedente familiar e sexo. 9,2\% apresentava diabetes mellitus e este foi associado ao estado civil, idade e pressão sistólica no momento da avaliação. Conclusões. Há uma população trabalhadora informal com elevada carga de adoecimento e fatores de risco que evidenciam a necessidade de políticas públicas e programas voltados especificamente para seus riscos e estilos de vida.

Palavras-chave: Setor informal, hipertensão, diabetes mellitus, mercado de trabalho. 
Hipertensión arterial, diabetes mellitus y factores de riesgo cardiovasculares en

trabajadores de una plaza de mercado de un municipio de Antioquia, Colombia, 2017-2018

\section{Introducción}

Las enfermedades no transmisibles (ENT) son cada vez más frecuentes a nivel mundial lo cual implica un aumento del gasto de bolsillo de las personas así como del gasto público en salud. Esto es especialmente importante para las enfermedades cardiovasculares las cuales se consideran la principal causa de defunción en el mundo con 17 millones de muertes anuales (1-3).

Según la Organización Mundial de la Salud (OMS) el 40\% de las personas mayores de 25 años en el mundo sufre de hipertensión arterial (HTA) y a sus complicaciones se les atribuyen 9,4 millones de muertes anuales (4,5). Adicionalmente, entre los factores identificados que aumentan el riesgo de sufrir HTA se destacan el alto consumo de sal o de grasa, la inactividad física, la ingesta deficiente de frutas y verduras, el sobrepeso y la obesidad, el consumo nocivo de alcohol, el estrés psicológico, los determinantes socioeconómicos como bajos ingresos económicos, bajo nivel de escolaridad y un inadecuado acceso a la atención sanitaria $(2,6)$.

Con respecto a la diabetes mellitus (DM) se prevé que para el año 2025 esta afecte alrededor de 350 millones de personas a nivel mundial (7). Su aumento ha sido atribuido a los estilos de vida sedentarios con factores de riesgo como el consumo de tabaco, el abuso de bebidas alcohólicas y una mala alimentación. Estos factores son considerados "condiciones de riesgo de la sociedad" y a su vez se relacionan con el desarrollo de sobrepeso y obesidad cuya presencia se asocia con el desarrollo de la DM y otras ENT (8).

Para el año 2013, el 5\% y 19\% de la población colombiana mayor de 45 años tenía DM e HTA, respectivamente, lo cual corresponde al $32 \%$ y $92 \%$ de todos los casos reportados para ese año (9). Además, dentro de los factores de riesgo presentes en adultos colombianos se estima una media de índice de masa corporal (IMC) para ambos sexos de $26.2 \mathrm{~kg} / \mathrm{m}$.; una prevalencia de sobrepeso y obesidad de $59 \%$ y $22 \%$, respectivamente; tabaquismo activo en mayores de 15 años para 2015 de $16 \%$ y $6 \%$ para hombres y mujeres, respectivamente; y $64 \%$ de la inactividad física (10).

Es preciso mencionar que el trabajo informal comprende entre la mitad y tres cuartos de los empleos no relacionados con la agricultura en los países en desarrollo (11). La condición de informalidad genera un entorno de vulnerabilidades para el individuo y su familia lo que se caracterizan por una alta carga laboral con baja remuneración económica y falta de aseguramiento. Estos factores asociados a otras vulnerabilidades como la edad van a desencadenar una perturbación tanto de la salud física como mental y social $(11,12)$.

Otros estudios han identificado en la población trabajadora de plazas de mercado una serie de condiciones de inseguridad laboral que incluyen el trabajo infantil, la exposición a altas temperaturas, ruidos fuertes y el manejo de cargas pesadas, siendo las enfermedades osteomusculares las más diagnosticadas en esta población $(13,14)$. 
Partiendo de lo anterior, el objetivo de la investigación es determinar la prevalencia de hipertensión arterial y diabetes mellitus en los trabajadores de una plaza de mercado del municipio Ciudad Bolívar del Departamento de Antioquia, Colombia, así como su asociación con variables clínicas, sociodemográficas y de estilos de vida del grupo poblacional analizado.

\section{Metodología}

Enfoque. Estudio observacional, de tipo transversal, con intención analítica y fuente primaria de información.

Población. Se realizó un censo de información sociodemográfica y laboral a 194 trabajadores, a 165 de ellos se les preguntó por variables clínicas, como resultado 152 trabajadores presentaron datos completos para los dos componentes. Se excluyeron del análisis los trabajadores que no presentaron una o ambas mediciones, bien sea por no presentarse a la valoración médica o por no estar en el puesto de trabajo durante los tres días de recolección de información. Se abordó a cada trabajador en su puesto de trabajo y se le explicó las características y condiciones de la investigación. A quienes aceptaron y firmaron el consentimiento informado se les aplicó la encuesta de caracterización sociodemográfica y laboral con variables sociodemográficas, de hábitos y estilos de vida, laborales y de los puestos de venta y su entorno. Posteriormente el trabajador se desplazó hacia el lugar dispuesto en la plaza de mercado para realizársele la evaluación médico-laboral con una ficha médica de base. En el caso de los menores de edad se realizó la evaluación y toma de datos con previa toma de asentimiento informado del menor y consentimiento informado por parte de un adulto encargado.

Recolección de la información. El personal que recolectó los datos de las encuestas y los médicos que realizaron la valoración de los trabajadores recibieron previamente su estandarización mediante una prueba piloto con los instrumentos estructurados.

Análisis de la información. La información fue analizada en SPSS21® (licencia obtenida por la Universidad CES) en el cual se realizó análisis univariado y bivariado de los datos considerando un valor alfa del 5\% y un intervalo de confianza (IC) del 95\%. Se calcularon distribuciones de frecuencias y porcentajes para las variables cualitativas y los estadísticos descriptivos para las cuantitativas. Además se calcularon razones de prevalencias crudas (RP) con sus respectivos IC del 95\%.

\section{Resultados}

De los 194 participantes de la encuesta sociodemográfica el 57\% es mujer y el resto hombres. La mediana de edad fue de 43 años (Rq.21-55). Además, 7\% de los participantes tenía entre 14 y 17 años y $47 \%$ entre 45 y 82 años. Con respecto a la escolaridad la mediana de años de estudio fue de 7 (Rq.3-11). Adicionalmente, el 39\% refirió no tener pareja. Ver tabla 1. 
Con respecto a las variables económicas, la mitad de los trabajadores recibía ingresos mensuales de $\$ 500.000$ COP (Rq.240.000-750.000) siendo al rededor del doble para los hombres que las mujeres. Se evidencia que $73 \%$ recibía menos de un salario mínimo mensual legal vigente (SMMLV) lo que equivaldría a $\$ 737.717$ COP (246,18 USD a 2017). El 71\% de los participantes estaba afiliado al sistema general de seguridad social en salud (SGSSS) y el 12\% cotizaba para el sistema general de pensiones (SGP). Además, $57 \%$ al momento de la encuesta era cabeza de familia ( $45 \%$ de las mujeres y $74 \%$ de los hombres). Ver tabla 1.

Tabla 1. Distribución de frecuencias, porcentajes y estadísticos descriptivos de las variables sociodemográficas de los trabajadores participantes en el estudio. $\mathrm{N}=194$

\begin{tabular}{|c|c|c|c|c|}
\hline \multirow{2}{*}{ Variable } & \multirow{2}{*}{ Categoría } & Hombres & Mujeres & Total \\
\hline & & n (\%) & n $(\%)$ & n (\%) \\
\hline \multirow{2}{*}{ Estado civil } & Con pareja & $46(55,4)$ & $72(65,5)$ & $118(60,8)$ \\
\hline & Sin pareja & $37(44,6)$ & $38(34,5)$ & $75(38,9)$ \\
\hline \multirow{2}{*}{$\begin{array}{l}\text { Tipo de afiliación } \\
\text { al SGSSS }\end{array}$} & Subsidiado & $56(67,5)$ & $81(74,3)$ & $137(71,4)$ \\
\hline & Contributivo & $27(32,5)$ & $28(25,7)$ & $55(28,6)$ \\
\hline \multirow{2}{*}{ Cotiza al SGP } & $\mathrm{Si}$ & $16(19,3)$ & $7(6,5)$ & $23(12,0)$ \\
\hline & No & $67(80,7)$ & $101(93,5)$ & $168(88,0)$ \\
\hline \multirow{2}{*}{ Cabeza de familia } & $\mathrm{Si}$ & $62(73,8)$ & $49(44,5)$ & $111(57,2)$ \\
\hline & No & $22(26,2)$ & $61(55,5)$ & $83(42,8)$ \\
\hline \multirow{3}{*}{$\begin{array}{l}\text { Promedio de } \\
\text { ingresos mensuales } \\
\text { (smmlv) }\end{array}$} & Menos de 1 & $47(57,3)$ & $94(85,5)$ & $141(73,4)$ \\
\hline & Entre 1 y 2 & $31(37,8)$ & $15(13,6)$ & $46(24)$ \\
\hline & Más de 2 & $4(4,9)$ & $1(0,9)$ & $5(2,6)$ \\
\hline \multirow{4}{*}{$\begin{array}{l}\text { Dinero pagado en } \\
\text { salud en el último } \\
\text { mes }(C O P)^{*}\end{array}$} & 0 & $49(59,8)$ & $59(55,7)$ & $108(57,5)$ \\
\hline & $<50.000$ & $16(19,5)$ & $31(29,2)$ & $47(25)$ \\
\hline & $50.000<100.000$ & $8(9,8)$ & $11(10,4)$ & $19(10,1)$ \\
\hline & $>100.000$ & $9(11,0)$ & $5(4,7)$ & $14(7,5)$ \\
\hline \multicolumn{2}{|c|}{ Variable } & $\operatorname{Me}(\mathbf{R q})$ & Me (Rq) & $\operatorname{Me}(\mathrm{Rq})$ \\
\hline \multicolumn{2}{|l|}{ Edad } & $50(68)$ & $39(60)$ & $43(68)$ \\
\hline \multicolumn{2}{|c|}{ Escolaridad en años } & $5(10)$ & $10(6)$ & $7(8)$ \\
\hline \multicolumn{2}{|c|}{ Promedio de ingresos al mes } & $700.000(540.000)$ & $310.000(400.000)$ & $500.000(510.000)$ \\
\hline
\end{tabular}

SGSSS: Sistema General de Seguridad Social en Salud. SGP: Sistema General de Pensiones. SMMLV: Salario Mínimo Mensual Legal Vigente. COP: pesos colombianos. *1 USD = 3009,55 COP (17/12/2017).

Fuente: elaboración propia.

Los resultados indican que alrededor del $66 \%$ trabajaba entre 9 y 12 horas diarias, y un $12 \%$ trabajaba entre 13 y 18 horas diarias. Un $47 \%$ de los trabajadores laboraba entre 6 y 7 días por semana.

En cuanto a los hábitos y estilos de vida de los trabajadores, el $62 \%$ no realizaba ninguna actividad física y un $26 \%$ no tenía ninguna actividad recreativa en su tiempo libre siendo mayor la prevalencia en mujeres para ambos casos. Además, el 53\% refirió como actividad recreativa "ver televisión", el $72 \%$ de los trabajadores manifestó ser sedentario y poco activo, y el $60 \%$ 
consideró que su peso era adecuado. Un 25\% consumían licor y un 16\% tenía el hábito de fumar mientras que un $37 \%$ usaba el salero en la mesa y el $67 \%$ prefería los "fritos" como método de cocción de los alimentos. Ver tabla 2.

Tabla 2. Distribución de frecuencias, porcentajes y estadísticos descriptivos de las variables de hábitos y estilos de vida de los trabajadores participantes en el estudio. N=194

\begin{tabular}{|c|c|c|c|c|c|}
\hline \multirow{2}{*}{\multicolumn{2}{|c|}{ Variable }} & \multirow{2}{*}{ Categoría } & Hombres & Mujeres & Total \\
\hline & & & n (\%) & n (\%) & n (\%) \\
\hline \multicolumn{3}{|c|}{ Ninguna actividad deportiva } & $46(54,8)$ & $75(68,2)$ & $121(62,4)$ \\
\hline \multicolumn{3}{|c|}{ Ninguna actividad recreativa } & $18(21,4)$ & $32(29,1)$ & $50(25,8)$ \\
\hline \multicolumn{3}{|c|}{ Ver televisión como actividad recreativa } & $43(51,2)$ & $59(53,6)$ & $102(52,6)$ \\
\hline \multirow{2}{*}{$\begin{array}{l}\text { Nivel de } \\
\text { actividad física }\end{array}$} & \multicolumn{2}{|c|}{ Poco activo / sedentario } & $58(69,9)$ & $81(73,6)$ & $139(72)$ \\
\hline & \multicolumn{2}{|c|}{ Activo / muy activo } & $25(30,1)$ & $29(26,4)$ & $54(28)$ \\
\hline \multirow{4}{*}{$\begin{array}{l}\text { Autopercepción } \\
\text { de peso }\end{array}$} & \multicolumn{2}{|c|}{ Bajo peso } & $6(7,1)$ & $11(10,1)$ & $17(8,8)$ \\
\hline & \multicolumn{2}{|l|}{ Adecuado } & $57(67,9)$ & $58(53,2)$ & $115(59,6)$ \\
\hline & \multicolumn{2}{|l|}{ Sobrepeso } & $20(23,8)$ & $37(33,9)$ & $57(29,5)$ \\
\hline & \multicolumn{2}{|l|}{ Obesidad } & $1(1,2)$ & $3(2,8)$ & $4(2,1)$ \\
\hline \multirow{2}{*}{ Consumo de licor } & \multicolumn{2}{|l|}{$\mathrm{Si}$} & $28(33,3)$ & $20(18,2)$ & $48(24,7)$ \\
\hline & \multicolumn{2}{|l|}{ No } & $56(66,7)$ & $90(81,8)$ & $146(75,3)$ \\
\hline \multirow{3}{*}{$\begin{array}{l}\text { Frecuencia de } \\
\text { consumo de licor }\end{array}$} & \multicolumn{2}{|c|}{ Una vez a la semana } & $15(57,7)$ & $9(50,0)$ & $24(54,5)$ \\
\hline & \multicolumn{2}{|c|}{ Dos a tres veces por semana } & $7(26,9)$ & $4(22,2)$ & $11(25)$ \\
\hline & \multicolumn{2}{|c|}{ Más de tres veces por semana } & $4(15,4)$ & $5(27,8)$ & $9(20,5)$ \\
\hline \multicolumn{3}{|c|}{ Hábito de fumar - Si } & $19(22,6)$ & $12(11,0)$ & $31(16,1)$ \\
\hline \multicolumn{3}{|c|}{ Utiliza salero en la mesa - $\mathrm{Si}$} & $34(40,5)$ & $37(33,6)$ & $71(36,6)$ \\
\hline \multicolumn{3}{|c|}{ Frito como método de cocción favorito - Si } & $60(71,4)$ & $70(63,6)$ & $130(67,0)$ \\
\hline \multicolumn{3}{|c|}{ Variable } & Me (Rq) & Me (Rq) & Me (Rq) \\
\hline \multicolumn{3}{|c|}{ Horas de trabajo por día } & $11,5(3,7)$ & $11(3,0)$ & $11(0,0)$ \\
\hline \multicolumn{3}{|c|}{ Días de trabajo por semana } & $6(3,0)$ & $3(4,0)$ & $5(5,0)$ \\
\hline \multicolumn{3}{|c|}{ Antigüedad de años en el sector } & $12(22,0)$ & $5(16,25)$ & $8(18,0)$ \\
\hline
\end{tabular}

Fuente: elaboración propia.

En lo que tiene que ver con las condiciones clínicas evaluadas en los trabajadores se evidenció que un 9\% tenía diagnóstico previo de DM y un 21\% de HTA. El 31\% reportó que en su familia había al menos un miembro con diagnóstico de DM y el 41\% de HTA. Al momento de la evaluación se identificó que un 12\% de los trabajadores tenía valores de presión arterial sistólica por encima del valor normal y el 11\% tenía valores de presión arterial diastólica por encima del valor normal. Ver tabla 3. 
El 24\% y 15\% de los diagnosticados con HTA tenían valores de presión sistólica y diastólica por encima de 140 y $90 \mathrm{~mm} \mathrm{Hg}$ al momento de su evaluación, en relación con el 9\% y 10\% de quienes afirmaron no haber sido diagnosticados con estas patologías. Por último, el $44 \%$ de los trabajadores se percibe en sobrepeso y $17 \%$ en obesidad.

Tabla 3. Distribución de frecuencias, porcentajes y estadísticos descriptivos de las condiciones de salud de los trabajadores participantes en el estudio. $\mathrm{N}=152$

\begin{tabular}{|c|c|c|c|}
\hline \multirow{2}{*}{ Variable } & Hombres & Mujeres & Total \\
\hline & n (\%) & n (\%) & n (\%) \\
\hline AP de diabetes mellitus & $9(11,7)$ & $6(7,0)$ & $15(9,2)$ \\
\hline $\mathrm{AF}$ de diabetes mellitus & $18(23,4)$ & $32(37,2)$ & $50(30,7)$ \\
\hline AP de hipertensión arterial & $19(24,7)$ & $15(17,4)$ & $34(20,9)$ \\
\hline AF de hipertensión arterial & $27(35,1)$ & $40(46,5)$ & $67(41,1)$ \\
\hline AP de enfermedad coronaria & $3(3,9)$ & $4(4,7)$ & $7(4,3)$ \\
\hline AP de enfermedad cerebrovascular & $3(3,9)$ & $1(1,2)$ & $4(2,5)$ \\
\hline IMC de sobrepeso u obesidad & $54(69,2)$ & $46(53,5)$ & $100(61,0)$ \\
\hline Alteración de la agudeza visual & $35(47,3)$ & $32(40,0)$ & $67(43,5)$ \\
\hline Variable & Me (Rq) & Me (Rq) & Me (Rq) \\
\hline Índice de Masa Corporal & $26,56(4,93)$ & $26,22(7,81)$ & $26,33(6,43)$ \\
\hline PAS a la evaluación & $120(20,0)$ & $110(16,0)$ & $120(15,0)$ \\
\hline PAD a la evaluación & $80(15,0)$ & $70(12,0)$ & $80(12,0)$ \\
\hline
\end{tabular}

AP: antecedente personal, AF: antecedente familiar, PAS: presión arterial sistólica; PAD: presión arterial diastólica.

Fuente: elaboración propia.

Frente al análisis bivariado se identificaron asociaciones estadísticamente significativas entre el diagnóstico de Hipertensión arterial (HTA) y el estado civil "tener pareja", así como con quienes manifestaron el antecedente familiar de HTA. Para la primera comparación los trabajadores que tenían pareja presentaron una prevalencia de HTA 4,5 veces menor con respecto a quienes estaban solteros $(\mathrm{RP}=0,22$. $\mathrm{IC}=0,11-0,45)$. De igual forma, aquellos con antecedente familiar de HTA presentaron una prevalencia de esta condición 2,7 veces menor con respecto a quienes no lo tenían ( $\mathrm{RP}=0,37$. $\mathrm{IC}=0,17-0,80)$. Para características como el sexo (ser hombre) no realizar actividad deportiva o recreativa y ser sedentario, aunque no se evidencia una asociación estadísticamente significativa sí se presenta una mayor prevalencia de HTA en aquellos que cumplen con estos criterios por aparte. Ver tabla 4. 
Tabla 4. Análisis bivariado: asociación entre las principales variables cualitativas e hipertensión arterial. $\mathrm{N}=152$

\begin{tabular}{|c|c|c|c|c|c|c|}
\hline \multirow{3}{*}{ Variable } & \multirow{3}{*}{ Categoría } & \multicolumn{5}{|c|}{ Hipertensión arterial } \\
\hline & & Si & No & & & \\
\hline & & $\begin{array}{c}\mathbf{n} \\
(\%)\end{array}$ & $\begin{array}{c}n \\
(\%)\end{array}$ & Chi2 & $\begin{array}{l}\text { valor } \\
\text { p }\end{array}$ & $(\mathrm{IC} 95 \%)$ \\
\hline Sexo & Hombre & $\begin{array}{c}19 \\
(55,9)\end{array}$ & $\begin{array}{c}58 \\
(45,0)\end{array}$ & 1,288 & 0,256 & $\begin{array}{c}1,42 \\
(0,77-2,59)\end{array}$ \\
\hline $\begin{array}{l}\text { Cabeza de } \\
\text { familia }\end{array}$ & $\mathrm{Si}$ & $\begin{array}{c}21 \\
(61,8)\end{array}$ & $\begin{array}{c}76 \\
(58,9)\end{array}$ & 0,091 & 0,763 & $\begin{array}{c}1,10 \\
(0,59-2,04)\end{array}$ \\
\hline Estado civil & Con pareja & $\begin{array}{c}8 \\
(23,5)\end{array}$ & $\begin{array}{c}87 \\
(68,0)\end{array}$ & 21,875 & 0,000 & $\begin{array}{c}0,22 \\
(0,11-0,45)\end{array}$ \\
\hline \multicolumn{2}{|c|}{ No realiza ninguna actividad deportiva } & $\begin{array}{c}23 \\
(67,6)\end{array}$ & $\begin{array}{c}80 \\
(62,0)\end{array}$ & 0,367 & 0,545 & $\begin{array}{c}1,22 \\
(0,64-2,32)\end{array}$ \\
\hline \multicolumn{2}{|c|}{ No realiza ninguna actividad recreativa } & $\begin{array}{c}12 \\
(35,3)\end{array}$ & $\begin{array}{c}31 \\
(24,0)\end{array}$ & 1,758 & 0,185 & $\begin{array}{c}1,42 \\
(0,83-2,80)\end{array}$ \\
\hline \multirow{2}{*}{$\begin{array}{l}\text { Nivel de } \\
\text { actividad física }\end{array}$} & $\begin{array}{l}\text { Poco activo o } \\
\text { sedentario }\end{array}$ & $\begin{array}{c}27 \\
(79,4)\end{array}$ & $\begin{array}{c}92 \\
(71,9)\end{array}$ & \multirow{2}{*}{0,783} & \multirow{2}{*}{0,376} & $\begin{array}{c}1,39 \\
(0,66-2,96)\end{array}$ \\
\hline & Activo o muy activo & $\begin{array}{c}7 \\
(20,6)\end{array}$ & $\begin{array}{c}36 \\
(28,1)\end{array}$ & & & 1 \\
\hline \multirow{2}{*}{$\begin{array}{l}\text { Autopercepción } \\
\text { de peso }\end{array}$} & Adecuado/bajo & $\begin{array}{c}20 \\
(60,6)\end{array}$ & $\begin{array}{c}88 \\
(68,2)\end{array}$ & \multirow{2}{*}{0,685} & \multirow{2}{*}{0,408} & $\begin{array}{c}0,77 \\
(0,42-1,43)\end{array}$ \\
\hline & Sobrepeso/obesidad & $\begin{array}{c}13 \\
(39,4)\end{array}$ & $\begin{array}{c}41 \\
(31,8)\end{array}$ & & & 1 \\
\hline \multirow{2}{*}{$\begin{array}{l}\text { Afiliación al } \\
\text { SGSSS }\end{array}$} & Subsidiado & $\begin{array}{c}21 \\
(61,8)\end{array}$ & $\begin{array}{c}92 \\
(72,4)\end{array}$ & \multirow{2}{*}{1,461} & \multirow{2}{*}{0,227} & $\begin{array}{c}0,69 \\
(0,37-1,26)\end{array}$ \\
\hline & Contributivo & $\begin{array}{c}13 \\
(38,2)\end{array}$ & $\begin{array}{c}35 \\
(27,6)\end{array}$ & & & 1 \\
\hline \multicolumn{2}{|l|}{ Consume de licor } & $\begin{array}{c}5 \\
(14,7)\end{array}$ & $\begin{array}{c}33 \\
(25,6)\end{array}$ & 1,780 & 0,182 & $\begin{array}{c}0,57 \\
(0,23-1,36)\end{array}$ \\
\hline \multicolumn{2}{|l|}{ Hábito de fumar } & $\begin{array}{c}2 \\
(5,9)\end{array}$ & $\begin{array}{c}22 \\
(17,2)\end{array}$ & 2,721 & 0,099 & $\begin{array}{c}0,359 \\
(0,09-1,20)\end{array}$ \\
\hline \multicolumn{2}{|c|}{ Utiliza salero en la mesa } & $\begin{array}{c}13 \\
(38,2)\end{array}$ & $\begin{array}{c}48 \\
(37,2)\end{array}$ & 0,012 & 0,912 & $\begin{array}{c}1,04 \\
(0,56-1,91)\end{array}$ \\
\hline \multicolumn{2}{|c|}{ Antecedente familiar de HTA } & $\begin{array}{c}7 \\
(20,6)\end{array}$ & $\begin{array}{c}60 \\
(46,5)\end{array}$ & 7,470 & 0,006 & $\begin{array}{c}0,37 \\
(0,17-0,80)\end{array}$ \\
\hline \multicolumn{2}{|c|}{ Antecedente familiar de DM } & $\begin{array}{c}10 \\
(29,4)\end{array}$ & $\begin{array}{c}40 \\
(31,0)\end{array}$ & 0,032 & 0,858 & $\begin{array}{c}0,94 \\
(0,49-1,82)\end{array}$ \\
\hline
\end{tabular}

SGSSS: Sistema General de Seguridad Social en Salud, HTA: hipertensión arterial, DM: diabetes mellitus.

Fuente: elaboración propia.

Aunque las condiciones y características evaluadas con relación a la DM no presentaron asociaciones estadísticamente significativas, características como el ser hombre, cabeza de familia, estar soltero y tener el antecedente familiar de HTA al momento de la toma de datos muestran asociación con una mayor prevalencia de Diabetes Mellitus. Ver tabla 5. 
Tabla 5. Análisis bivariado: tablas cruzadas entre las principales variables cualitativas y diabetes. $\mathrm{N}=152$

\begin{tabular}{|c|c|c|c|c|c|c|}
\hline \multirow{3}{*}{ Variable } & \multirow{3}{*}{ Categoría } & \multicolumn{5}{|c|}{ Diabetes mellitus } \\
\hline & & $\mathbf{S i}$ & No & & & \\
\hline & & $\begin{array}{c}n \\
(\%)\end{array}$ & $\begin{array}{c}n \\
(\%)\end{array}$ & Chi2 & valor $p$ & (IC 95\%) \\
\hline Sexo & Hombre & $\begin{array}{c}9 \\
(60,0)\end{array}$ & $\begin{array}{c}68 \\
(45,9)\end{array}$ & 1,079 & 0,299 & $\begin{array}{c}1,68 \\
(0,63-4,49)\end{array}$ \\
\hline $\begin{array}{l}\text { Cabeza de } \\
\text { familia }\end{array}$ & $\mathrm{Si}$ & $\begin{array}{c}10 \\
(66,7)\end{array}$ & $\begin{array}{c}87 \\
(58,8)\end{array}$ & 0,351 & 0,553 & $\begin{array}{c}1,36 \\
(0,48-3,80)\end{array}$ \\
\hline Estado civil & Con pareja & $\begin{array}{c}4 \\
(26,7)\end{array}$ & $\begin{array}{c}91 \\
(61,9)\end{array}$ & 6,969 & 0,08 & $\begin{array}{c}0,26 \\
(0,09-0,77)\end{array}$ \\
\hline \multicolumn{2}{|c|}{ No realiza ninguna actividad deportiva } & $\begin{array}{c}9 \\
(60,0)\end{array}$ & $\begin{array}{c}94 \\
(63,5)\end{array}$ & 0,072 & 0,788 & $\begin{array}{c}0,87 \\
(0,33-2,34)\end{array}$ \\
\hline \multicolumn{2}{|c|}{ No realiza ninguna actividad recreativa } & $\begin{array}{c}5 \\
(33,3)\end{array}$ & $\begin{array}{c}38 \\
(25,7)\end{array}$ & 0,393 & 0,544 & $\begin{array}{c}1,39 \\
(0,51-3,85)\end{array}$ \\
\hline \multirow{2}{*}{$\begin{array}{l}\text { Nivel de } \\
\text { actividad física }\end{array}$} & $\begin{array}{l}\text { Poco activo o } \\
\text { sedentario }\end{array}$ & $\begin{array}{c}12 \\
(80,0)\end{array}$ & $\begin{array}{c}107 \\
(72,8)\end{array}$ & \multirow{2}{*}{0,383} & \multirow{2}{*}{0,761} & $\begin{array}{c}1,45 \\
(0,43-4,88)\end{array}$ \\
\hline & Activo o muy activo & $\begin{array}{c}3 \\
(20,0)\end{array}$ & $\begin{array}{c}40 \\
(27,2)\end{array}$ & & & 1 \\
\hline \multirow{2}{*}{$\begin{array}{l}\text { Autopercepción } \\
\text { de peso }\end{array}$} & Adecuado/bajo & $\begin{array}{c}8 \\
(53,3)\end{array}$ & $\begin{array}{c}100 \\
(68,0)\end{array}$ & \multirow{2}{*}{1,322} & \multirow{2}{*}{0,250} & $\begin{array}{c}0,57 \\
(0,22-1,49)\end{array}$ \\
\hline & Sobrepeso/obesidad & $\begin{array}{c}7 \\
(46,7)\end{array}$ & $\begin{array}{c}47 \\
(32,0)\end{array}$ & & & 1 \\
\hline \multirow{2}{*}{$\begin{array}{l}\text { Afiliación al } \\
\text { SGSSS }\end{array}$} & Subsidiado & $\begin{array}{c}11 \\
(73,3)\end{array}$ & $\begin{array}{c}102 \\
(69,9)\end{array}$ & \multirow{2}{*}{0,080} & \multirow{2}{*}{1,000} & $\begin{array}{c}1,17 \\
(0,39-3,49)\end{array}$ \\
\hline & Contributivo & $\begin{array}{c}4 \\
(26,7)\end{array}$ & $\begin{array}{c}44 \\
(30,1)\end{array}$ & & & 1 \\
\hline \multicolumn{2}{|l|}{ Consume de licor } & $\begin{array}{c}1 \\
(6,7)\end{array}$ & $\begin{array}{c}37 \\
(25,0)\end{array}$ & 3,228 & 0,196 & $\begin{array}{c}0,24 \\
(0,03-1,73)\end{array}$ \\
\hline \multicolumn{2}{|l|}{ Hábito de fumar } & $\begin{array}{c}2 \\
(13,3)\end{array}$ & $\begin{array}{c}22 \\
(15,0)\end{array}$ & 0,029 & 1,000 & $\begin{array}{c}0,89 \\
(0,21-3,68)\end{array}$ \\
\hline \multicolumn{2}{|c|}{ Utiliza salero en la mesa } & $\begin{array}{c}7 \\
(46,7)\end{array}$ & $\begin{array}{c}54 \\
(36,5)\end{array}$ & 0,603 & 0,438 & $\begin{array}{c}1,46 \\
(0,56-3,84)\end{array}$ \\
\hline \multicolumn{2}{|c|}{ Antecedente familiar de HTA } & $\begin{array}{c}3 \\
(20,0)\end{array}$ & $\begin{array}{c}64 \\
(43,2)\end{array}$ & 3,039 & 0,081 & $\begin{array}{c}0,36 \\
(0,11-1,22)\end{array}$ \\
\hline \multicolumn{2}{|c|}{ Antecedente familiar de DM } & $\begin{array}{c}4 \\
(26,7)\end{array}$ & $\begin{array}{c}46 \\
(31,1)\end{array}$ & 0,128 & 1,000 & $\begin{array}{c}0,82 \\
(0,28-2,46)\end{array}$ \\
\hline
\end{tabular}

SGSSS: Sistema General de Seguridad Social en Salud. HTA: hipertensión arterial. DM: diabetes mellitus.

Fuente: elaboración propia.

Con respecto a la autopercepción de peso por cada 10 trabajadores que se consideraban en sobrepeso u obesidad y tenían HTA se identificaron 7 que tenían esta patología y se consideran en peso adecuado, comportamiento similar al que se evidencia entre esta variable con el diagnóstico de DM. 
Al evaluar la clasificación de los participantes a partir del IMC quienes estaban en condición de sobrepeso u obesidad presentaban una mayor prevalencia de DM $(\mathrm{RP}=2,13$. IC95\% $=0,62-7,32)$ y de HTA $(R P=2,154$. IC95\%=1,00-4,65) que aquellos que no presentaban esta condición. Esta última asociación fue estadísticamente significativa y deja ver como por cada trabajador que no presentaban condición de sobre peso/obesidad y tenía diagnóstico de HTA se observaron 2,15 trabajadores en condición de sobrepeso/obesidad (datos no mostrados).

Al comparar la autopercepción de sobrepeso y obesidad con la misma categoría obtenida por el valor del IMC mientras el $49 \%$ de quienes tienen un IMC en rango de normalidad se considera en sobrepeso, sólo el 5\% de quienes están en sobrepeso según su rango de normalidad se consideraba en sobrepeso (datos no mostrados).

Con respecto a la presencia de HTA y la DM según la edad de los trabajadores se identificaron diferencias estadísticamente significativas que dejan ver cómo eran mayores aquellos que tenían el diagnóstico de alguna de estas patologías con respecto a quienes no. También se observaron diferencias estadísticamente significativas con el dinero pagado en el último mes en gastos de salud, las horas de trabajo al día y la presión sistólica al momento de la evaluación, siendo mayor en todos los casos para quienes tenían el antecedente de HTA y DM tanto en hombres como mujeres. Aunque no se identificaron diferencias estadísticamente significativas sí se evidencia la tendencia a ser menor la escolaridad, los ingresos promedios al mes, las horas de trabajo al día, en ambos grupos de comparación (HTA y DM) y mayor el IMC y la presión arterial diastólica al momento de la evaluación. Ver tabla 6. 
Tabla 6. Análisis bivariado: tablas cruzadas entre las principales variables cuantitativas HTA y DM. $\mathrm{N}=152$

\begin{tabular}{|c|c|c|c|c|c|c|}
\hline \multirow{3}{*}{ Variable } & \multicolumn{3}{|c|}{ Hipertensión arterial } & \multicolumn{3}{|c|}{ Diabetes mellitus } \\
\hline & \multirow{2}{*}{$\frac{\text { Si }}{\operatorname{Me}(\mathbf{R q})}$} & \multirow{2}{*}{$\begin{array}{c}\text { No } \\
\text { Me }(\mathbf{R q}) \\
\end{array}$} & \multirow{2}{*}{ Valor p } & \multirow{2}{*}{$\begin{array}{c}\text { Si } \\
\text { Me (Rq) }\end{array}$} & \multirow{2}{*}{$\frac{\text { No }}{\operatorname{Me~(Rq)}}$} & \multirow{2}{*}{ Valor p } \\
\hline & & & & & & \\
\hline Edad (años) & 61 & 42 & 0,0000 & 59 & 43 & 0,000 \\
\hline Escolaridad (años) & 5 & 8 & 0,051 & 4,5 & 7 & 0,124 \\
\hline Ingresos mensuales promedio & 450.000 & 600.000 & 0,988 & 500.000 & 600.000 & 0,922 \\
\hline Horas de trabajo por día & 10 & 11 & 0,132 & 8,5 & 11 & 0,154 \\
\hline Antigüedad en el oficio & 19,5 & 10 & 0,009 & 7,5 & 10 & 0,260 \\
\hline Índice de masa corporal & 27,19 & 26,88 & 0,169 & 27,20 & 26,83 & 0,242 \\
\hline $\begin{array}{l}\text { Presión sistólica al momento } \\
\text { de la evaluación }\end{array}$ & 130 & 115 & 0,000 & 130 & 120 & 0,010 \\
\hline $\begin{array}{l}\text { Presión diastólica al momento } \\
\text { de la evaluación }\end{array}$ & 80 & 80 & 0,062 & 82,5 & 80,0 & 0,071 \\
\hline
\end{tabular}

Todas las variables presentan valores $p$ en prueba de Shapiro-Wilk $<0,05$.

Fuente: elaboración propia.

\section{Discusión}

Aunque este tipo de diseño limita la confirmación de causalidad es de especial valor como sustento para el planteamiento de hipótesis para futuras investigaciones de orden analítico y causal en poblaciones trabajadoras en las que se presentan diversas condiciones de empleabilidad laboral. Es el caso de quienes ejercen sus labores en las plazas de mercado tanto de los municipios de Colombia como de otros países de la región.

Si bien la mayor proporción de trabajadores que hicieron parte de la muestra estaba en edad laboral, una cantidad considerable estaba por encima de los 65 años superando la edad actual de jubilación para los hombres y mujeres en Colombia. Ante esto se debe agregar que tres cuartas partes de los trabajadores reportaron que sus ingresos mensuales eran menos de un SMMLV. La situación se complejiza si se tiene en cuenta que casi la mitad de los trabajadores tenía personas que dependían económicamente de sus ingresos y una escasa proporción de los trabajadores cotizaba a pensiones. Lo anterior expone la inseguridad laboral a la que está expuesta la población trabajadora de adultos mayores. Así, su carga de enfermedad puede verse complicada por la precariedad de empleos, los cuales podrían incluso considerarse como empleos de subsistencia porque "deben trabajar de día para comer de noche" (15).

La diversa economía de una plaza de mercado como sitio de trabajo da paso a una amplia variabilidad en las condiciones laborales y de vida que no deben desconocerse $(15,16)$. Esta investigación identificó que los trabajadores de la plaza de mercado de Ciudad Bolívar, uno de 
los municipios del Suroeste antioqueño, se exponen a una alta carga laboral que se ve reflejada en largas jornadas de trabajo y limitados días de descanso a la semana asociadas a mayor estrés físico, psicológico y una mayor susceptibilidad para el desarrollo de enfermedades no transmisibles como la HTA y la DM.

Para esta población trabajadora los estilos de vida adoptados por fuerza de su realidad implican el sedentarismo dado por un tiempo muy limitado para el deporte y la recreación a partir de la necesidad de cumplir con sus trabajos junto a la carga económica de sus hogares. Frente a esto se deben considerar otros factores culturales que puedan influir en hechos como que la mitad tuvieran como actividad recreativa ver televisión y que una alta proporción de trabajadores dentro de sus preferencias tuviera el consumo de alimentos fritos. De forma global, estos datos deberían ser una alerta para que los agentes promotores de salud desarrollen estrategias de promoción y prevención que impacten los espacios habitados por esta población con largas jornadas laborales y pocos espacios de esparcimiento.

Con respecto a los resultados de las variables clínicas, la alta prevalencia de HTA como de DM evidenciada se encuentra $15 \%$ por encima de la prevalencia nacional para HTA y $12 \%$ para la de DM (datos no mostrados en los resultados) $(9,17)$. Al comparar por sexos tanto el diagnóstico de DM como el de HTA fueron más frecuentes en hombres que en mujeres, así como el consumo de licor y el hábito de fumar cigarrillo.

Llama la atención la autopercepción de peso de los trabajadores que tiende a subvalorar su condición real y más en aquellos con comorbilidades como HTA y DM. Así, mientras que del $50 \%$ de quienes estaban en peso adecuado se percibían en sobrepeso, sólo el 5\% de quienes estaban en sobrepeso u obesidad se consideraban en esta categoría. A pesar de las limitaciones del estudio estos resultados deberían llevar a los agentes promotores de salud a considerar el papel que puede tener la autopercepción de las personas sobre su cuerpo y el impacto de las acciones de promoción de salud y prevención de la enfermedad.

La obesidad se ha identificado como un factor de riesgo para el desarrollo tanto de DM como de HTA, coronariopatías, accidentes cerebrovasculares, ciertos tipos de cáncer (2). La prevalencia de sobrepeso y obesidad en los sujetos de este estudio es bastante similar a la reportada a nivel nacional al igual que el estilo de vida sedentario; sin embargo, destaca que el hábito de fumar para esta población se encuentra alrededor de $6 \%$ más tanto para hombres como para mujeres, persistiendo un consumo mayor para los hombres (10).

La literatura es clara en mostrar los beneficios de intervenir en los factores de riesgo. Para el caso de la obesidad se ha demostrado que su manejo puede evidenciar, sino un retraso en el desarrollo de la enfermedad, al menos sí una regresión parcial de la misma con mejoría en los síntomas y su control (18). Otras medidas recomendadas son la asesoría nutricional, el incentivo a la actividad física y la farmacoterapia oportuna en el control de situaciones de riesgo con 
énfasis en la educación para el paciente y su familia, lo cual brinda herramientas para que con el apoyo familiar pueda tener un mejor resultado de la situación $(18,19)$.

Uno de los ejemplos de políticas públicas en Latinoamérica es el impuesto de 1 peso por litro de bebida azucarada implementado en México que, incluso estando por debajo de las recomendaciones establecidas por la OMS, ha evidenciado un cambio favorable en la conducta de la demanda especialmente en las poblaciones de menor estratos socioeconómicos (20-22). Este caso muestra la importancia de involucrar a toda la sociedad en la formulación y el desarrollo de políticas públicas para las ENT alrededor de las patologías con un enfoque en el ciclo vital de las personas y con la reorientación del sistema de salud haciendo hincapié en la prevención y control mediante una atención primaria con el reconocimiento de los determinantes sociales y un enfoque individualizado a partir de este, tal y como lo recomienda la $28^{a}$ Conferencia Sanitaria Panamericana (23).

Algunos autores han demostrado que entre más abajo se encuentren las personas en su escala social y a medida que esto represente una menor autonomía van a estar más limitados para satisfacer sus necesidades básicas y esto los va a predisponer más a enfermedades dentro de las que se destacan las crónicas no transmisibles (24). Aunque predominan muchas situaciones en contra, el trabajo informal representa acceso a la autonomía laboral en aquellos que deciden ser los encargados de su propio negocio. En comparación con otras personas del mismo nivel socioeconómico que trabajen en calidad de empleado, esto representa un aspecto a favor para su ya amplio ambiente de riesgo (11).

Una importante proporción de los encuestados lleva más de 5 años trabajando en este oficio, lo cual es el reflejo de una forma de estabilidad laboral que puede representar el empleo informal a pesar de las inseguridades mencionadas anteriormente. Frente a esto es de destacar que la población trabajadora informal hace parte característicamente de la población de bajos recursos así esto no lo establezca su definición (25). Tal situación expone a los trabajadores al llamado "síndrome de estatus" que para un país como Colombia con su inequidad hace que estas personas estén expuestos a un mayor riesgo y una mayor carga de la enfermedad no propiamente por su posición socioeconómica, sino por el impacto que pueden tener los determinantes intermedios en la salud del trabajador con respecto a otros individuos de mayor posición $(12,26)$.

\section{Conclusión}

Las autoridades sanitarias y de la administración municipal deben adelantar acciones dirigidas a los trabajadores de plazas de mercado que superen las supeditadas a las políticas públicas y programas que tratan el riesgo cardiovascular de la población como un todo. Además de estrategias de prevención y control de las enfermedades crónicas no transmisibles en favor de un impacto desde los determinantes sociales de la salud haciendo énfasis en el adecuado abordaje desde el primer nivel de atención (6). Esta población debe representar un reto para las respectivas entidades dada su poca disponibilidad de tiempo fuera de sus sitios de trabajo, los riesgos propios de la informalidad laboral, la falta de aseguramiento y una fuerte influencia cultural que impide 
el reconocimiento de sus factores de riesgo como lo es la obesidad, tal y como lo reflejan los resultados de este trabajo.

\section{Reconocimientos}

Los autores agradecen a la Facultad de Medicina de la Universidad CES por la aprobación del proyecto y a la población trabajadora de la plaza de mercado del municipio Ciudad Bolívar, Antioquia, que accedieron a participar voluntariamente en esta investigación. Igualmente, agradecen el apoyo y acompañamiento de la Secretaría de Salud del municipio, al Hospital E.S.E La María y a la Facultad de Salud Pública de la Universidad de Antioquia, Colombia.

\section{Consideraciones éticas}

Estudio aprobado por el Comité Institucional de Ética en Seres Humanos de la Universidad CES mediante Acta 123 código de proyecto Ae162 acorde con el cumplimiento de lo establecido en la resolución 8430 de 1993 de Colombia y en la Declaración de Helsinki de 1975. Investigación clasificada como de riesgo mínimo.

\section{Financiación}

Este proyecto fue financiado con recursos de los investigadores, recursos de la Secretaría de Salud de Ciudad Bolívar y el Hospital E.S.E La María, así como también de la Facultad de Medicina de la Universidad CES.

\section{Conflictos de interés}

Ninguno de los autores reporta conflicto de interés asociado al desarrollo y presentación de este trabajo de investigación.

\section{Referencias}

1. Jamison D, Etienne C. Prefacio. Las dimensiones económicas de las enfermedades no transmisibles en América Latina y el Caribe. Washington DC: OPS; 2017. p.V-VI. http://iris.paho.org/xmlui/bitstrea $\mathrm{m} /$ handle/123456789/33994/9789275319055-spa.pdf?sequence=1\&isAllowed=y

2. Organización Mundial de la Salud (OMS). Informe sobre la situación mundial de las enfermedades no transmisibles. Ginebra; 2014. http://apps.who.int/iris/bitstream/handle/10665/149296/WHO_NMH_ NVI_15.1_spa.pdf;jsessionid=B2E3BF92C4778B75162AD8474655B658? sequence=1

3. World Health Organization (WHO). Causes of death 2008: Data sources and methods. Geneva; 2011. ht tps://www.who.int/healthinfo/global_burden_disease/cod_2008_sources_methods.pdf

4. Lim SS, Vos T, Flaxman AD, Danaei G, Shibuya K, Adair-Rohani H, et al. A comparative risk assessment of burden of disease and injury attributable to 67 risk factors and risk factor clusters in 21 regions, 
Hipertensión arterial, diabetes mellitus y factores de riesgo cardiovasculares en

trabajadores de una plaza de mercado de un municipio de Antioquia, Colombia, 2017-2018

1990-2010: A systematic analysis for the Global Burden of Disease Study 2010. Lancet. 2012 Dec 15;380(9859):2224-2260. http://www.ncbi.nlm.nih.gov/pubmed/23245609

5. World Health Organization (WHO). Global status report on noncommunicable disease 2010. Geneva; 2011. http://www.who.int/about/licensing/copyright_form/en/index.html

6. World Health Organization (WHO). A Global Brief on Hypertension. Geneva; 2013. www.who.int/about/ licensing/copyright_form/en/index.html

7. Farkouh ME, Rayfield EJ, Fuster V. Diabetes and cardiovascular disease. In: Fuster V, Harrington R, Narula J, Eapen Z (eds). Hurst's The Heart, 14e. New York: McGraw-Hill Education; 2017. http://a ccessmedicine.mhmedical.com/content.aspx?aid=1154846897

8. Blanco A, Jacoby E, Monteiro M, Caixeta R, Smith B, Grajeda R, et al. Factores de riesgo en las Américas: orígenes de la carga. En: Las dimensiones económicas de las enfermedades no transmisibles en América Latina y el Caribe. Washington DC: OPS; 2017. p. 25-33. http://iris.paho.org/xmlui/bitstre am/handle/123456789/33994/9789275319055-spa.pdf?sequence=1\&isAllowed=y

9. Ministerio de Salud y Protección Social (MinSalud). Análisis de Situación de Salud Colombia. Bogotá; 2016. https://www.minsalud.gov.co/sites/rid/Lists/BibliotecaDigital/RIDE/VS/ED/PSP/asis-colomb ia-2016.pdf

10. World Health Organization (WHO). Global Health Observatory data repository. World Health Organization; 2018. http://apps.who.int/gho/data/node.home

11. Lund F. Social protection and the informal economy: Linkages and good practices for poverty reduction and empowerment. In: Promoting pro-poor growth: Social protection. Geneva: OECD; 2009. p.69-88. http://www.oecd.org/development/povertyreduction/43280700.pdf

12. Vélez C, Escobar M, Pico M. Determinantes sociales de la salud y el trabajo informal. Rev Costarr Salud Pública. 2013;22(2):156-162. https://www.researchgate.net/publication/263474018_Determin antes_sociales_de_la_salud_y_el_trabajo_informal

13. Gómez-Palencia IP, Castillo-Ávila IY, Banquez-Salas AP, Castro-Ortega AJ, Lara-Escarlate HR. Condiciones de trabajo y salud de vendedores informales estacionarios del mercado de Bazurto, en Cartagena. Rev Salud Pública. 2012;14:448-459. https://www.scielosp.org/article/rsap/2012.v14n3/ $448-459 /$

14. Briceño L, Pinzón AM. Trabajo infantil en una plaza de mercado de Bogotá, Colombia. Rev Salud Pública. 2005;7(1):26-38. http://www.scielo.org.co/pdf/rsap/v7n1/v7n1a3.pdf

15. Garzón-Duque MO, Cardona-Arango MD, Rodríguez-Ospina FL, Segura-Cardona AM. Informalidad y vulnerabilidad laboral: aplicación en vendedores con empleos de subsistencia. Rev Saude Publica. 2017;51:1-17. https://scielosp.org/pdf/rsp/2017.v51/89/es

16. International Labour Organization. Informal economy. 2018. http://www.ilo.org/ilostat-files/Documen ts/description_IFL_EN.pdf

17. Aschner P. Epidemiología de la diabetes en Colombia. Av en Diabetol. 2010;26(2):71-134. https://link inghub.elsevier.com/retrieve/pii/S1134323010620054

18. American Diabetes Association. Obesity management for the treatment of type 2 diabetes. Diabetes Care. 2016;39(Suppl 1):S47-S51. http://www.ncbi.nlm.nih.gov/pubmed/26696681 
19. American Diabetes Association. Lifestyle management: standards of medical care in diabetes. Diabetes Care. 2018 Jan;41(Suppl 1):S38-S50. http://www.ncbi.nlm.nih.gov/pubmed/29222375

20. Organización Panamericana de la Salud (OPS). Los impuestos a los refrescos y a las bebidas azucaradas como medida de salud pública. https://www.paho.org/mex/index.php?option=com_content\&view=a rticle\&id=627:los-impuestos-refrescos-bebidas-azucaradas-medida-salud-publica\&Itemid=499

21. Álvarez-Sánchez C, Contento I, Jiménez-Aguilar A, Koch P, Gray HL, Guerra LA, et al. Does the Mexican sugar-sweetened beverage tax have a signaling effect? ENSANUT 2016. PLoS One. 2018 Aug 22;13(8):e0199337. https://doi.org/10.1371/journal.pone.0199337

22. Colchero MA, Salgado JC, Unar-Munguía M, Molina M, Ng S, Rivera-Dommarco JA. Changes in prices after an excise tax to sweetened sugar beverages was implemented in Mexico: Evidence from urban Areas. Nugent RA. PLoS One. 2015 Dec 14;10(12):e0144408. https://dx.plos.org/10.1371/jo urnal.pone. 0144408

23. Organización Panamericana de la Salud (OPS). Estrategia para la prevención y el control de las enfermedades no transmisibles, 2012-2025. Washington DC; 2012. https:/www.paho.org/hq/dmdo cuments/2012/CSP28-9-s.pdf

24. Marmot MG. Status syndrome. JAMA. 2006;296(4):395-396. https://doi.org/10.1001/jama.296.4.395

25. International Labour Organization. Resolution concerning decent work and the informal economy; 2002. https://www.ilo.org/public/english/standards/relm/ilc/ilc90/pdf/pr-25res.pdf

26. Marmot M. Status syndrome: A challenge to medicine. JAMA. 2006;295(11):1304-1307. http://dx.do i.org/10.1001/jama.295.11.1304 